\title{
Correlation of Surgical Times with Laparoscopic Live Donor Kidney Transplant Outcomes
}

\author{
Sertac Cimen ${ }^{1}$, Sanem Guler ${ }^{2}$, Ian Alwayn ${ }^{1}$, Joseph Lawen ${ }^{3}$, Bryce Kiberd ${ }^{4}$ \\ ${ }^{1}$ The Division of General Surgery, Dalhousie University, Queen Elizabeth II Health Sciences Centre, Halifax, Canada \\ ${ }^{2}$ Atlantic Centre for Transplantation Research, Dalhousie University, Halifax, Canada \\ ${ }^{3}$ Department of Urology, Dalhousie University, Queen Elizabeth II Health Sciences Centre, Halifax, Canada \\ ${ }^{4}$ Faculty of Medicine, Dalhousie University, Queen Elizabeth II Health Sciences Centre, Halifax, Canada \\ Email: Bryce.Kiberd@dal.ca
}

Received September 14, 2013; revised October 14, 2013; accepted October 21, 2013

Copyright (C) 2013 Sertac Cimen et al. This is an open access article distributed under the Creative Commons Attribution License, which permits unrestricted use, distribution, and reproduction in any medium, provided the original work is properly cited.

\begin{abstract}
Most studies revealed that ischemic time has substantial role in occurrence of delayed graft function (DGF) after deceased donor kidney transplantation. However, less is known about the potential impact of surgical times on early outcomes following live donor kidney transplantation. A retrospective cohort of 189 consecutive laparoscopic live donor kidney transplant (LDKT) recipients from January 2006 to August 2012 was analyzed to reveal the impact of pneumoperitoneum time (PT) and anastomosis time (AT) on donor and recipient length of hospital stay and early graft function (EGF). DGF was observed in $13(6.8 \%)$ patients while slow graft function (SGF) was seen in $27(14 \%)$ of the recipients. The median AT was 28 minutes (interquartile range 23, 35 minutes). AT was associated with DGF (Odds Ratio [OR] 1.044, per minute, $95 \%$ CI 1.007, 1.082, $\mathrm{p}=0.018$ ). Median recipient length of hospital stay was 8 (interquartile range 7,11 ) days. Every 13.5 minutes of longer AT was associated with 1 extra day in hospital. The median PT was 180 minutes (interquartile range 144, 234 minutes). PT was associated with both DGF (OR 1.013 per minute, 95\% CI $1.005,1.021, \mathrm{p}=0.001$ ) and SGF (OR 1.009 per minute, 95\% CI 1.002, 1.016, p=0.016). Every extra hour of PT was associated with 0.42 more days in hospital for the donor. Surgical times may be underestimated variables in dictating use of hospital resources. The effect of surgical times on long term hard outcomes entails further study.
\end{abstract}

Keywords: Delayed Graft Function; Slow Graft Function; Anastomosis Time; Pneumoperitoneum Time; Kidney Transplantation

\section{Introduction}

Since its description in 1995 laparoscopic live donor nephrectomy has replaced open donor nephrectomy at many transplant centres [1]. Multiple studies have shown that laparoscopic nephrectomy is considerably gentler for the donor with less postoperative pain, shorter length of hospital stay and improved cosmesis [2,3]. However some studies have shown that the pneumoperitoneum used during laparoscopic donor nephrectomy procedure might impair renal blood flow and prolonged pneumoperitoneum time (PT) can increase the oxidative stress and subsequently cause subtle renal tissue injury [4-6].

In line with this hypothesis, several investigators have reported slower early post-transplant function for laparoscopically retrieved (vs. open) grafts as measured by poor early graft function (EGF) rates $[7,8]$. This is important because even mild to moderate early graft dysfunction can have a negative impact on long-term graft survival [9].

Studies investigating EGF after kidney transplant from living donors (regardless of the procurement technique) identified prolonged anastomosis time (AT) as another risk factor for the occurrence of poor EGF [10].

In the present study we critically assessed the correlation of PT and AT with the indicators of EGF after laparoscopic live donor kidney transplant (LDKT) surgeries.

\section{Materials and Methods}

Consecutive laparoscopic LDKT donors and recipients who underwent surgery between January 2006 and $\mathrm{Au}-$ gust 2012 were identified. Data were extracted retrospectively from the hospital's electronic records. Approval for this study was obtained from our institution's re- 
search ethics board.

All donors were admitted the day before surgery. All of the laparoscopic donor nephrectomy procedures were performed by transperitoneal route except for one which was done by retroperitoneoscopic approach. Intravenous fluids, mannitol and furosemide were given as required to help prevent pneumoperitoneum-induced oliguria. The renal vessels were controlled individually by using endoscopic vascular stapler. The graft was extracted through either Pfannensteil incision using an endobag or handport. PT was recorded in each case as the time between its initiation and extraction of the graft.

All recipients received induction immunosuppression with either basiliximab or antithymocyte globulin (ATG) and all of them were given methylprednisolone sodium succinate $500 \mathrm{mg}$ intraoperatively. ATG was reserved for those who were repeat transplant recipients or highly sensitized. All received pneumocystis pneumonia and gastric ulcer prophylaxis. For patients already on a proton pump inhibitor pre-transplant, this agent was continued instead of starting ranitidine. Cytomegalovirus (CMV) prophylaxis with valganciclovir was used when the donor was seropositive in a seronegative recipient or when the recipient was induced by ATG. AT was recorded in each case as the time between the end of the cooling period and reperfusion of the kidney. All patients subsequently received were put on oral tacrolimus, mycophenolate and a prednisone tapering dose.

Data extracted for recipients included age, gender, weight, body mass index (BMI), calculated panel reactive antibodies (cPRA), Human Leukocyte Antigen mismatch (HLA MM) status, cause of end stage renal disease (ESRD), diabetes mellitus (DM), AT (minutes), serum creatinine (Cr) pre-transplant and on postoperative day (POD) 5, delayed graft function (DGF), slow graft function (SGF) and duration of hospital stay.

Data extracted for the donors included age, gender, weight, BMI, pre-donation and discharge $\mathrm{Cr}$, duration of hospital stay, PT, side of the retrieved kidney and retrieval technique (laparoscopy/conversion to open donor nephrectomy).

Statistical data are presented as mean and standard deviation (SD) for continuous normally distributed variables, median and interquartile range for continuous variables not normally distributed and percentages for dichotomous variables. Variables associated with DGF (need for dialysis within the first week after kidney transplant) were examined by logistic regression analysis. Since there were a small number of events for DGF, a detailed multivariable linear regression analysis could not be completed. Instead, a univariable logistic regression analysis was done. A binary logistic regression analysis was also performed on whether that patient had SGF which was defined as POD5 creatinine $\geq 265 \mu \mathrm{mol} / \mathrm{L}$ without need of dialysis in the first week post-transplant. A multivariable linear regression analysis was performed for recipient POD5 $\mathrm{Cr}$ and duration of hospital stay using both recipient and donor variables. However since $>40 \%$ of values were missing for cPRA, this variable was not included in the analysis. A multivariable linear regression analysis was performed for the donor duration of hospital stay using donor variables only. Variables that were significant at $p$ $<0.1$ in a univariable analysis were included into the multivariable models. Overall significance was assumed at $\mathrm{p}<0.05$ level. Statistical analysis was performed by IBM SPSS Statistics software version 20.0 (New York, US).

\section{Results}

There were 209 consecutive live donor kidney transplant operations. Patients undergoing open nephrectomy were excluded $(\mathrm{n}=16)$ as well as laparoscopic operations without recorded warm ischemic times $(n=4)$, leaving 189 subjects for evaluation.

Population descriptors are displayed in Tables $\mathbf{1}$ and $\mathbf{2}$ for recipients and donors respectively. Mean ages of the recipients and donors were similar. Donors were more likely female and recipients were more likely men.

Table 1. Recipient descriptors and key results.

\begin{tabular}{|c|c|}
\hline \multicolumn{2}{|c|}{ Recipient } \\
\hline Age years mean (SD) & $45(14)$ \\
\hline Male & $120(63 \%)$ \\
\hline Body Weight kg Mean (SD) & $80(19)$ \\
\hline $\begin{array}{c}\text { Body Mass Index kg/m² } \\
\text { Mean (SD) }\end{array}$ & $28(6)$ \\
\hline HLA Mismatch Mean (SD) & $3.1(1.9)$ \\
\hline $\begin{array}{c}\text { ESRD } \\
\text { DM } \\
\text { Glomerulonephritis } \\
\text { Interstitial Disease } \\
\text { PCKD } \\
\text { Other }\end{array}$ & $\begin{array}{l}31(16 \%) \\
79(42 \%) \\
24(13 \%) \\
28(15 \%) \\
27(14 \%)\end{array}$ \\
\hline $\begin{array}{c}\text { AT minutes } \\
\text { Mean (SD) } \\
\text { Median (IQR) }\end{array}$ & $\begin{array}{c}31(14) \\
28(23,35)\end{array}$ \\
\hline $\begin{array}{c}\text { Creatinine } \mu \mathrm{mol} / \mathrm{L} \\
\text { Admission } \\
\text { Mean (SD) } \\
\text { Median (IQR) } \\
\text { POD 5 }\end{array}$ & $\begin{array}{c}715(263) \\
666(521,885)\end{array}$ \\
\hline $\begin{array}{c}\text { Mean (SD) } \\
\text { Median (IQR) }\end{array}$ & $\begin{array}{c}198(187) \\
135(100,195)\end{array}$ \\
\hline $\begin{array}{c}\text { Change day } 0 \text { to } 5 \\
\text { Mean (SD) } \\
\text { Median (IQR) }\end{array}$ & $\begin{array}{c}519(268) \\
490(344,678)\end{array}$ \\
\hline $\begin{array}{l}\text { Days in hospital } \\
\text { Mean (SD) } \\
\text { Median (IQR) }\end{array}$ & $\begin{array}{c}10.8(8.2) \\
8(7,11)\end{array}$ \\
\hline DGF & $13(6.8 \%)$ \\
\hline SGF & $27(14 \%)$ \\
\hline
\end{tabular}


Table 2. Live donor descriptors and key results.

\begin{tabular}{cc}
\hline & Donor \\
\hline Age years mean (SD) & $43(11)$ \\
Male & $67(36 \%)$ \\
Body Weight kg mean (SD) & $75(15)$ \\
Body Mass Index kg/m² & $27(6)$ \\
Conversion & $6(3 \%)$ \\
Left & $103(55 \%)$ \\
PT minutes & \\
Mean (SD) & $196(66)$ \\
Median (IQR) & $180(144,234)$ \\
Creatinine $\mu$ mol/L & $72(14)$ \\
Admission & $111(20)$ \\
Discharge & $39(13)$ \\
Difference & \\
Days in hospital & $5.8 \pm 1.7$ \\
Mean (SD) & $6(5,6)$ \\
Median (IQR) &
\end{tabular}

Median and interquartile ranges (IQR) are included for AT (Figure 1), PT (Figure 2), days in hospital and recipient POD5 $\mathrm{Cr}$ since these outcomes were not normally distributed.

DGF was observed in $13(6.8 \%)$ of patients. Significant variables associated with DGF in a binary logistic regression analysis were AT (Odds Ratio [OR] 1.044, per minute, $95 \%$ CI 1.007, 1.082, $\mathrm{p}=0.018$ ), PT (OR 1.013 per minute, $95 \%$ CI $1.005,1.021, \mathrm{p}=0.001)$ and pretransplant Cr (OR 1.003 per $\mu \mathrm{mol} / \mathrm{L}, 95 \%$ CI 1.001, 1.004 , $\mathrm{p}=0.007)$. AT and PT were significantly correlated $(\mathrm{r}=$ $0.34, \mathrm{p}<0.001)$ however pre-transplant creatinine was not correlated with AT or PT.

SGF was observed in $27(14 \%)$ of patients. Significant variables associated with SGF in a binary logistic regression analysis were PT (OR 1.009 per minute, 95\% CI $1.002,1.016, \mathrm{p}=0.016)$, recipient male (OR 9.6, 95\% CI $1.2,76, \mathrm{p}=0.032)$ and pre-transplant $\mathrm{Cr}(\mathrm{OR} 1.002$ per $\mu \mathrm{mol} / \mathrm{L}, 95 \%$ CI $1.000,1.004, \mathrm{p}=0.014)$. AT was significant $(p=0.019)$ in the univariable analysis but not in the multivariable model. No other variables were significant.

In a multivariable linear regression analysis, POD5 $\mathrm{Cr}$ was associated with recipient weight (Beta coefficient $1.7 \mu \mathrm{mol} / \mathrm{L}$ per $\mathrm{kg}, 95 \% \mathrm{CI} 0.3,3.1, \mathrm{p}=0.018$ ), recipient pre-transplant $\mathrm{Cr}(0.17 \mu \mathrm{mol} / \mathrm{L}$ per $\mu \mathrm{mol} / \mathrm{L}, 95 \%$ CI 0.07 , $0.26, \mathrm{p}=0.001)$ and AT $(3.5 \mu \mathrm{mol} / \mathrm{L}$ per $\min , 95 \% \mathrm{CI}$ $1.6,5.3, \mathrm{p}<0.001)$. HLA MM, donor gender, donor predonation $\mathrm{Cr}$ and PT were significant $(\mathrm{p}<0.10)$ in the univariate analysis and were included in the model but were not independent predictors. Therefore every 10 longer minutes of AT was associated with a $\mathrm{Cr} 35 \mu \mathrm{mol} / \mathrm{L}$ higher on POD5.

For associations with recipient stay in hospital, AT (Beta coefficient 0.073 days per minute, 95\% CI 0.017 ,

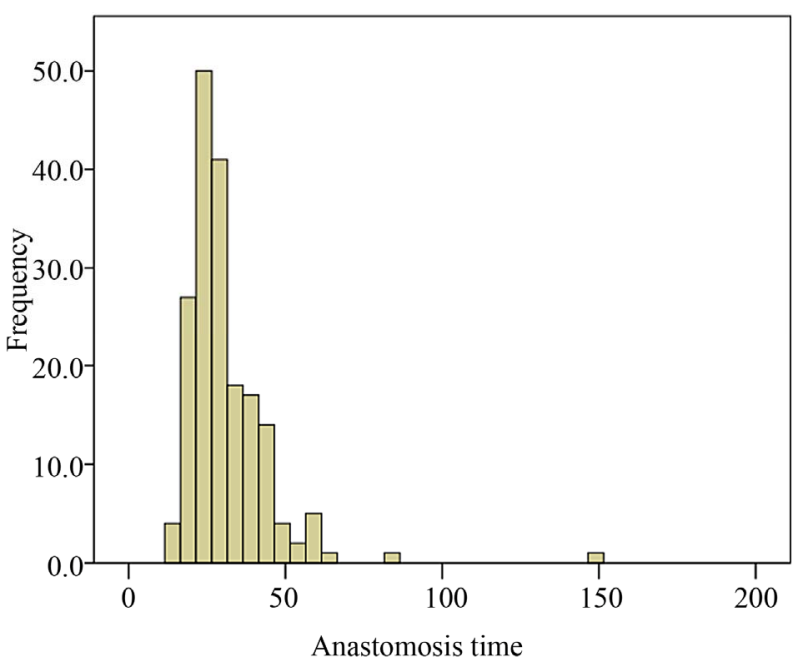

Figure 1. Distribution of anastomosis time among recipient population of the study.

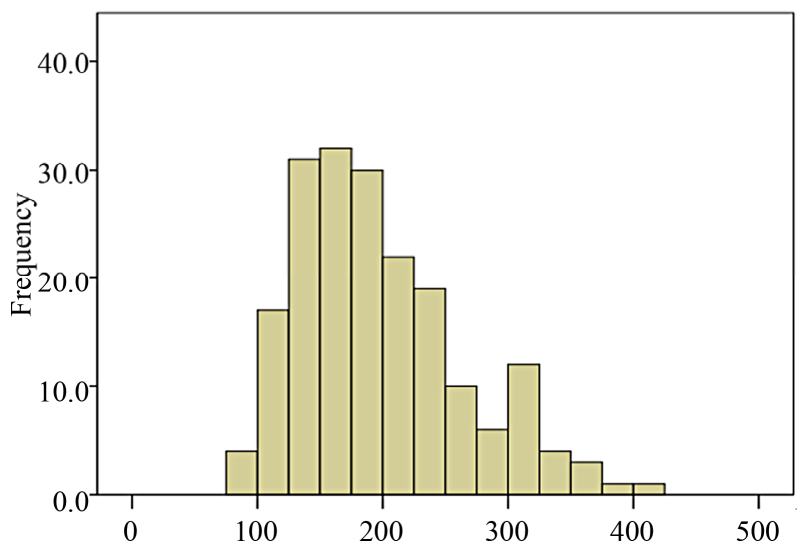

Figure 2. Distribution of pneumoperitoneum time among donor population of the study.

$0.13, \mathrm{p}=0.011)$, HLA MM (0.48 days per MM, $95 \% \mathrm{CI}$ $0.1,0.9, \mathrm{p}=0.014)$, recipient pre-transplant $\mathrm{Cr}(0.004$ days per $\mu \mathrm{mol} / \mathrm{L}, 95 \% \mathrm{CI} 0.001,0.007, \mathrm{p}=0.008)$ and donor age (0.074 days per year, $95 \%$ CI $0.005,0.14, \mathrm{p}=$ 0.035 ) were significant in a multivariable linear regression analysis. PT was significant in a univariable analysis $(p=0.002)$ but was not independently significant in the multivariable model. All other variables were not significant. Therefore every 13.5 minutes of longer AT was associated with 1 extra day in hospital.

For associations with donor stay in hospital, only PT (Beta coefficient 0.007 days per min, 95\% CI 0.003, 0.011, $\mathrm{p}<0.001)$ and conversion to open surgery (1.4 days, $95 \%$ CI $0.05,2.7, p=0.42$ ) were significant. Every extra hour of PT was associated with 0.42 more days in hospital for the donor.

\section{Discussion}

The purpose of this study was to examine the effect of 
AT and PT on short term outcomes in laparoscopic LDKT. AT was associated with DGF, higher recipient POD5 serum $\mathrm{Cr}$, and longer recipient stays in hospital. PT was also associated with DGF as well as longer donor hospital stay. Theoretically longer AT and PT could be associated with ischemic injury, longer stays in hospital and possibly poor long term function. We also examined SGF as SGF has been associated with poor long term graft survival in deceased donor kidney transplantation (DDKT) $[11,12]$. Surprisingly PT but not AT was associated with SGF in our study.

In a recent study regarding impact of AT on the indicators of EGF after DDKT, AT associated with DGF [13]. This report also showed that every 5 minutes of longer AT added 1 extra day to the duration of recipient hospital stay after DDKT. In our current study 13.5 minutes of longer AT leaded to the same result. This significant difference can be explained by the fact that while the former report investigated DDKTs, our current study involved LDKTs.

Sharma et al. also evaluated the factors causing DGF after LDKT [14]. In this study, kidneys were predominately procured by standard open approach. Total (retrieval and anastomosis) WIT was found as a major determinant of DGF. Duration of hospital stay was significantly longer in the patient group with DGF when compared with the non-DGF patient group.

In contrast to Sharma et al., our study was exclusively on laparoscopic kidney retrieval. Since almost $30 \%$ of the retrieval WIT data were missing, we were unable to calculate the total WIT. On the other hand, WIT associated with retrieval is generally quite short and the median and mean values in our study were both 5 minutes.

Hellegering et al. investigated the determinants of poor EGF and showed that prolonged ( $>45$ minutes) total WIT (retrieval and anastomosis) was one of the predictors of poor EGF in either open or laparoscopic LDKT [15]. Brennan et al. also examined the risk factors for poor EGF in LDKTs performed after either open or laparoscopic donor nephrectomy [10]. In this study WIT emerged as an independent risk factor for poor EGF, however their WIT included the retrieval WIT.

Conversely Tyson et al. studied solely on laparoscopic LDKTs [16]. These authors did not find a correlation between WIT and poor EGF. Nevertheless they only examined the WIT associated with retrieval but not anastomosis.

Abreu et al. studied laparoscopically retrieved kidneys [17]. Although their cohort was relatively small $(n=100)$ and underpowered, they found that total preservation time was more important than AT with regard to graft function. In this study, total preservation time was the sum of total WIT and the cold ischemia time. This study highlights the importance of consistently defining and re- cording all surgical times.

Some experimental studies indicated that combination of prolonged $\mathrm{CO}_{2}$ pneumoperitoneum and ischemia during laparoscopic kidney retrieval may increase the risk of early postoperative graft dysfunction $[4,18]$. In line with these findings, it has been shown that recipients of laparoscopically procured kidneys had higher short-term serum $\mathrm{Cr}$ compared to recipients of kidneys procured by open technique $[7,19]$.

Abreu and coworkers examined the impact of PT and $\mathrm{CO}_{2}$ absorption on early graft function however they did not find any correlation [17]. We did not analyze mean $\mathrm{CO}_{2}$ absorption or pneumoperitoneum pressures. Nevertheless, as a general principle pneumoperitoneum pressures are rarely higher than $15 \mathrm{mmHg}$ during laparoscopic donor nephrectomy procedures. We investigated the impact of PT on EGF and found that it correlated significantly with both DGF and SGF.

Johnson et al. reported that length of hospital stay after laparoscopic donor nephrectomy was significantly longer in cases with prolonged operating time [20]. They ascribed this finding to the impact of prolonged surgery time on other organ systems of the donor. In our analysis, 1 hour of longer PT added 0.42 more days to the hospital stay in the donor.

The limitations of our study are its retrospective nature in addition to missing cPRA, retrieval WIT and pneumoperitoneum pressure data. We did not examine the long-term outcomes such as graft and patient survival as our sample size is quite small. Given the intercorrelation between PT and AT and the relatively small number of events for DGF and SGF, we are not able to predict their independent contributions to early outcomes confidently.

In summary our findings highlight the need for a standardized terminology and accurate documentation of all surgical times. Acknowledging the importance at surgical times is the first step towards finding innovative ways to minimize times and improve the outcomes. Larger studies with longer follow-up periods are needed to examine the impact of AT and PT on long-term patient and graft outcomes.

\section{REFERENCES}

[1] L. E. Ratner, L. J. Ciseck, R. G. Moore, F. G. Cigarroa, H. S. Kaufman and L. R. Kavoussi, "Laparoscopic Live Donor Nephrectomy," Transplantation, Vol. 60, No. 9, 1995, pp. 1047-1049.

[2] T. L. Merlin, D. F. Scott, M. M. Rao, et al., "The Safety and Efficacy of Laparoscopic Live Donor Nephrectomy: A Systematic Review," Transplantation, Vol. 70, No. 12, 2000, pp. 1659-1666.

http://dx.doi.org/10.1097/00007890-200012270-00001

[3] N. F. Kok, W. Weimar, I. P. Alwayn and J. N. Ijzermans, "The Current Practice of Live Donor Nephrectomy in 
Europe," Transplantation, Vol. 82, No. 7, 2006, pp. 892897. http://dx.doi.org/10.1097/01.tp.0000235511.19629.0d

[4] G. Akbulut, C. Polat, F. Aktepe, et al., "The Oxidative Effect of Prolonged $\mathrm{CO}_{2}$ Pneumoperitoneum on Renal Tissue of Rats," Surgical Endoscopy, Vol. 18, No. 9, 2004, pp. 1384-1388. http://dx.doi.org/10.1007/s00464-003-9114-9

[5] E. J. Hazebroek, R. W. de Bruin, N. D. Bouvy, et al., "Long-Term Impact of Pneumoperitoneum Used for Laparoscopic Donor Nephrectomy on Renal Function and Histomorphology in Donor and Recipient Rats," Annals of Surgery, Vol. 237, No. 3, 2003, pp. 351-357.

http://dx.doi.org/10.1097/01.SLA.0000055272.96210.A0

[6] E. J. Hazebroek, R. W. de Bruin, N. D. Bouvy, et al., "Short-Term Impact of Carbon Dioxide, Helium and Gasless Laparoscopic Donor Nephrectomy on Renal Function and Histomorphology in Donor and Recipient," Surgical Endoscopy, Vol. 16, No. 2, 2002, pp. 245-251. http://dx.doi.org/10.1007/s00464-001-8169-8

[7] C. Troppmann, D. B. Ormond and R. V. Perez, "Laparoscopic (vs Open) Live Donor Nephrectomy: A UNOS Database Analysis of Early Graft Function and Survival," American Journal of Transplantation, Vol. 3, No. 10, 2003, pp. 1295-1301.

http://dx.doi.org/10.1046/j.1600-6143.2003.00216.x

[8] J. M. Nogueira, C. B. Cangro, J. C. Fink, et al., "A Comparison of Recipient Renal Outcomes with Laparoscopic versus Open Live Donor Nephrectomy," Transplantation, Vol. 67, No. 5, 1999, pp. 722-728. http://dx.doi.org/10.1097/00007890-199903150-00014

[9] J. M. Nogueira, A. Haririan, S. C. Jacobs, et al., "The Detrimental Effect of Poor Early Graft Function after Laparoscopic Live Donor Nephrectomy on Graft Outcomes," American Journal of Transplantation, Vol. 9, No. 2, 2009, pp. 337-347. http://dx.doi.org/10.1111/j.1600-6143.2008.02477.x

[10] T. V. Brennan, C. E. Freise, T. F. Fuller, A. Bostrom, S. J. Tomlanovich and S Feng, "Early Graft Function after Living Donor Kidney Transplantation Predicts Rejection But Not Outcomes," American Journal of Transplantation, Vol. 4, No. 6, 2004, pp. 971-979. http://dx.doi.org/10.1111/j.1600-6143.2004.00441.x

[11] N. Smail, J. Tchervenkov, S. Paraskevas, et al., "Impact of Early Graft Function on 10 Year Graft Survival in Recipients of Kidneys from Standard or Expanded Criteria Donors," Transplantation, Vol. 96, No. 2, 2013, pp. 176181. http://dx.doi.org/10.1097/TP.0b013e318297443b
[12] A. Humar, E. M. Johnson, W. D. Payne, et al., "Effect of Initial Slow Graft Function on Renal Allograft Rejection and Survival," Clinical Transplantation, Vol. 11, No. 6, 1997, pp. 623-627.

[13] K. Marzouk, J. Lawen, I. Alwayn and B. A. Kiberd, "The Impact of Vascular Anastomosis Time on Early Kidney Transplant Outcomes," Transplantation Research, Vol. 2, No. 8, 2013. http://dx.doi.org/10.1186/2047-1440-2-8

[14] A. K. Sharma, S. L. Tolani, G. L. Rathi, P. Sharma, H. Gupta and R. Gupta, "Evaluation of Factors Causing Delayed Graft Function in Live Related Donor Renal Transplantation," Saudi Journal of Kidney Diseases and Transplantation, Vol. 21, No. 2, 2010, pp. 242-245.

[15] J. Hellegering, J. Visser, H. J. Kloke, et al., "Deleterious Influence of Prolonged Warm Ischemia in Living Donor Kidney Transplantation," Transplantation Proceedings, Vol. 44, No. 5, 2012, pp. 1222-1226. http://dx.doi.org/10.1016/j.transproceed.2012.01.118

[16] M. Tyson, E. Castle, P. Andrews, et al., "Early Graft Function after Laparoscopically Procured Living Donor Kidney Transplantation," Journal of Urology, Vol. 184, No. 4, 2010, pp. 1434-1439. http://dx.doi.org/10.1016/j.juro.2010.06.013

[17] S. C. Abreu, D. A. Goldfarb, I. Derweesh, et al., "Factors Related to Delayed Graft Function after Laparoscopic Live Donor Nephrectomy," Journal of Urology, Vol. 171 No. 1, 2004, pp. 52-57. http://dx.doi.org/10.1097/01.ju.0000098819.79442.ce

[18] W. Khoury, K. Jakowlev, A. Fein, H. Orenstein, R. Nakache and A. A. Weinbroum, "Renal Apoptosis Following Carbon Dioxide Pneumoperitoneum in a Rat Model," Journal of Urology, Vol. 180, No. 4, 2008, pp. 1554-1558. http://dx.doi.org/10.1016/j.juro.2008.06.008

[19] L. E. Ratner, L. R. Kavoussi, P. G. Schulam, J. S. Bender, T. H. Magnuson and R. Montgomery, "Comparison of Laparoscopic Live Donor Nephrectomy versus the Standard Open Approach," Transplantation Proceedings, Vol. 29, No. 1-2, 1997, pp. 138-139. http://dx.doi.org/10.1016/S0041-1345(96)00037-1

[20] E. M. Johnson, M. J. Remucal, K. J. Gillingham, R. A. Dahms, J. S. Najarian and A. J. Matas, "Complications and Risks of Living Donor Nephrectomy," Transplantation, Vol. 64, No. 8, 1997, pp. 1124-1128. http://dx.doi.org/10.1097/00007890-199710270-00007 